\title{
El negocio electrónico como estrategia de marketing
}

The electronic business as a marketing strategy

\author{
Jorge Martín Hernández - Mendoza a, Sandra Luz Hernández - Mendoza b, Edgar Olguín - \\ Guzmán c
}

\begin{abstract}
:
The impact presented today by the use of the internet around the world has been really overwhelming, in one way or another everyone uses this tool for various purposes and / or conducts business online, what we know as E-commerce or commerce electronic. In particular, small and medium-sized enterprises (PyMEs), commercial and services, require the use of these technologies that open their possibilities for growth and solid development, by increasing and strengthening their capabilities that allow them to strengthen their market position and consolidate its competitiveness This article describes a marketing strategy proposal, it arises as a concern to concentrate the key elements involved in the establishment of SME business strategies, supported by Internet-based e-commerce tools.
\end{abstract}

Keywords:

E-commerce, internet, strategy, business.

\section{Resumen:}

El impacto que presenta hoy en día el uso del internet en todo el mundo ha sido realmente arrollador, de una u otra forma todos recurren a esta herramienta con fines diversos y/o realizan negocios en línea, lo que conocemos como E-commerce o comercio electrónico. En particular, las pequeñas y medianas empresas (PyMEs), comerciales y de servicios, requieren del uso de estas tecnologías que abra sus posibilidades de crecimiento y sólido desarrollo, al incrementar y fortalecer sus capacidades que les permita fortalecer su posición en el mercado y consolidar su competitividad. El presente artículo describe una propuesta de estrategia mercadológica, surge como una inquietud para concentrar los elementos claves que intervienen en el establecimiento de estrategias de negocios de las PyMEs, apoyados en las herramientas de comercio electrónico basadas en Internet.

\section{Palabras Clave:}

Comercio electrónico, internet, estrategia, negocio.

\section{Introducción}

La economía actual muestra grandes cambios con respecto a la conocida hasta hace unos pocos años, como la tendencia hacia un mercado más competitivo, especializado, global y afianzado en Internet.
La combinación de las tecnologías de información y comunicación basadas en Internet y los procesos de negocios, han originado nuevos conceptos de comercialización con enfoque electrónico.

Las micros y pequeñas empresas, más flexibles, en general, en sus planteamientos que las medianas y

\footnotetext{
a Profesor Tiempo Completo de la Escuela Superior de Tepeji del Río. Universidad Autónoma del Estado de Hidalgo, Av. del Maestro \#41 Col. Noxtongo 2da Sección, C.P. 42854. ORCID:0000-0002-9262-4712, Email: jomar_hm@hotmail.com

b Profesor-Asignatura del Instituto de Ciencias Económico-Administrativas. Universidad Autónoma del Estado de Hidalgo. Área Académica de Turismo-Campus ICEA-La Concepción, km 2.5, San Juan Tilcuatla Municipio de San Agustín Tlaxiaca Hidalgo. C.P. 42161. ORCID: 0000-0002-2022-3135, e-mail: sandrahm@uaeh.edu.mx

a Profesor Tiempo Completo del Instituto de Ciencias Básicas e Ingeniería. Universidad Autónoma del Estado de Hidalgo, Ciudad del Conocimiento, Carretera Pachuca-Tulancingo Km. 4.5 Col. Carboneras, Mineral de la Reforma Hidalgo, C.P. 42184. ORCID:0000-00029003-6511, Email: eolguin@uaeh.edu.mx
} 
grandes empresas, pueden disponer aquí de un modelo de negocio nuevo, capaz de generar comercio electrónico rentable.

En México, aproximadamente el $98 \%$ de los establecimientos son micro, pequeñas y medianas empresas (SIEM, 2007), que presentan una gran capacidad de adaptación y crecimiento, y constituyen un factor crítico de estabilidad económica y social. Sin embargo ante el actual escenario económico de globalización, estas empresas enfrentan numerosos obstáculos y rezagos que limitan su capacidad productiva y que sólo podrán superarse mediante el uso correcto de las tecnologías de información orientadas al comercio electrónico.

En particular, las pequeñas y medianas empresas (PyMEs), comerciales y de servicios, requieren del uso de estas tecnologías que abra sus posibilidades de crecimiento y sólido desarrollo, al incrementar y fortalecer sus capacidades que les permita fortalecer su posición en el mercado y consolidar su competitividad [1][2].

\section{Generalidades de las PyMEs}

La actual globalización en nuestro país requiere que las empresas sean competitivas y generadoras de empleos. La pequeña y mediana empresa (PyME) constituye una de las grandes oportunidades para los países que, como México, quieren competir y exportar. El criterio adoptado por la Secretaría de Hacienda y Crédito Público, cataloga a las pequeñas y medianas empresas como aquellas cuyos ingresos acumulables en el ejercicio inmediato anterior no sean superior a veinte millones de pesos, pero tampoco inferiores a millón y medio de pesos; con esto el contribuyente podrá determinar fácilmente si su empresa es pequeña o mediana, o bien rebasa esta clasificación.

En la tabla 1, se muestra la clasificación, con datos proporcionados por Nacional Financiera y autorizados por su Consejo Directivo en diciembre de 2012 y publicados en el Diario Oficial de la Federación, para los sectores industrial, comercial y de servicios, y que es el mismo criterio que utiliza la Secretaría de Economía.

\begin{tabular}{|c|c|c|c|}
\hline \multirow{3}{*}{ TAMAÑO } & \multicolumn{3}{|c|}{ SECTOR } \\
\hline & \multicolumn{3}{|c|}{$\begin{array}{l}\text { CLASIFICACIÓN SEGÚN EL NÚMERO DE } \\
\text { EMPLEADOS }\end{array}$} \\
\hline & INDUSTRIA & COMERCIO & SERVICIOS \\
\hline Micro & De 0 a 10 & De 0 a 10 & $\mathrm{De} 0$ a 10 \\
\hline Pequeña & De 11 a 50 & De 11 a 30 & De 11 a 50 \\
\hline Mediana & De 51 a 250 & De 31 a 100 & De 51 a 100 \\
\hline Grande & Más de 250 & Más de 100 & Más de 100 \\
\hline
\end{tabular}

Fuente: Nacional Financiera, 2012.

\section{Las tecnologías de información y comunicación en la competitividad de las PyMES}

Un factor importante que define la presencia de las diferentes empresas en el escenario internacional y ubica a los países más allá de sus fronteras es la revolución tecnológica.

El aprendizaje tecnológico ha sido una pieza clave de la innovación y, por ende, de la competitividad. La tecnología y la habilidad para crearla y manejarla se han convertido en parte esencial del proceso competitivo, siendo éstas un factor necesario para ganar mercados o para conservarlos.

Sin duda, uno de los principales activos de las sociedades actuales son los servicios de comunicación o las Tecnologías de la Información y Comunicación (TIC), y la capacidad para acceder y ofrecer este tipo de servicios refleja su grado de avance y desarrollo tecnológico, así como su nivel competitivo en un escenario globalizado.

En la última década, los procesos de apertura comercial han orillado a los países y sus gobiernos a implementar políticas para fomentar el desarrollo y la investigación tecnológica; como resultado de esto, las sociedades han tenido acceso a mejores y más eficientes servicios de comunicación.

\section{Las PyMEs y el comercio electrónico}

Las PyMEs son el sector económico que más empleos genera y que más rápidamente crece en México. Son clave para acercar los productos al consumidor final y para dotar de insumos a las grandes empresas y cadenas comerciales. Sin embargo, la mayoría se encuentra rezagada tecnológicamente. Mientras que las grandes empresas siguen invirtiendo fuertemente para agilizar sus sistemas de administración y construir una presencia en el Internet, las pequeñas se encuentran en fase rudimentaria debido a la falta de capitales, de estímulos o simplemente de información.

De acuerdo con INEGI, en México, a principios del nuevo milenio, menos del $15 \%$ de las PyMEs cuenta con el apoyo de una computadora y apenas el $14 \%$ tiene soporte de especialistas en sistemas. El gran reto es vincular a estas empresas a las nuevas tecnologías. Así, un tema sobresaliente y actual, es la inversión en tecnología por parte de las empresas. El análisis del desarrollo tecnológico permite conocer las actividades 
que son más propensas al uso de la tecnología (CONCANACO, 2017: p. 47).

Muchas PyMEs ya comienzan a ser arrastradas por el cambio. Sus grandes clientes les exigen procesos tecnológicos básicos como enviar listas de precios o pedidos por la red, en otras palabras, empiezan a requerir herramientas a las que no están acostumbradas. Gracias a las nuevas herramientas de cómputo, las PyMEs pueden realizar el control de sus inventarios, llevar la contabilidad del negocio y comunicarse con proveedores y clientes, de manera bastante sencilla.[3]

De acuerdo a datos de INEGI, en el sector comercio, el tamaño de las empresas es directamente proporcional al uso de la tecnología informática en su relación con clientes y proveedores, en procesos técnicos, y en mejoramiento de procesos; no así en procesos administrativos, donde las diferencias de tamaño de la empresa no muestran variantes significativas (CONCANACO, 2017: p. 48).

En la tabla 2, se muestra el bajo uso de la tecnología en las PyMEs destacando la utilización del internet

\begin{tabular}{|c|c|c|c|c|}
\hline \multicolumn{5}{|c|}{$\begin{array}{c}\text { UTILIZACIÓN DEL INTERNET EN LOS PROCESOS DE } \\
\text { EMPRESAS COMERCIALES }\end{array}$} \\
\hline & 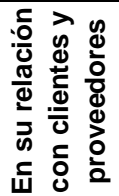 & 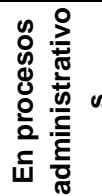 & 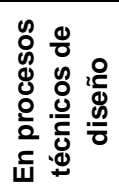 & 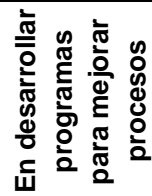 \\
\hline Micro & 4.1 & 6.9 & 2.5 & 2.2 \\
\hline Pequeña & 60.3 & 83.6 & 34.7 & 34.5 \\
\hline Mediana & 74.3 & 92.5 & 47.3 & 48.1 \\
\hline Grande & 79.8 & 94.6 & 55.8 & 66.3 \\
\hline
\end{tabular}

Tabla 2. Establecimientos comerciales que utilizaron equipo informático e Internet en alguno de sus procesos (\%). Fuente: INEGI, Censo Económico 2014.

Julio Villasante, socio de B-Nexus y Vicepresidente del Grupo de Trabajo de Proveedores de Tecnología del Comité Mexicano de Comercio Electrónico, afirma que el tema de la tecnología ya aparece como inquietud entre pequeños y medianos empresarios. Si bien por ahora son pocas las que están haciendo el cambio, el auge del comercio electrónico está sensibilizando a muchas.

Si bien es importante que las PyMEs comiencen a construir una presencia en Internet, lo mejor es avanzar con paso lento, para evitar gastos inútiles, comenzando por operaciones básicas para realizar operaciones como enviar y recibir órdenes y pedidos. Un segundo paso para entrar al comercio electrónico, es comenzar a intercambiar información a través de la red. Se puede empezar por colocar productos en catálogos electrónicos. Desde 1999, la Asociación Mexicana de Estándares para el Comercio Electrónico (AMECE) cuenta con un catálogo electrónico donde todos sus socios, cerca de 18 mil empresas, pueden subir sus productos sin costo alguno.

En una tercera etapa, el empresario pequeño puede abrir su propia página en Internet o su tienda virtual.

Puede ser que en un principio no suceda un aumento espectacular de las ventas, pero aquellas empresas que se encaminen hacia el comercio electrónico estarán cortando rápidamente la brecha de competitividad que las separa de las grandes corporaciones. [4]

Muchas empresas pequeñas se resisten al cambio porque consideran que implica fuertes inversiones. Lo cierto es que gracias a los múltiples planes de crédito que ya ofrece el mercado, hoy en día las computadoras están prácticamente al alcance de cualquier negocio.

La revolución del comercio electrónico está más cerca que nunca. Dentro de unos años, entre el 30 al $60 \%$ del comercio mundial se realizará a través de la red y aquellas empresas que no empiecen a construir una presencia en el Internet, podrían no sólo perder oportunidades sino a la larga quedarse fuera del mercado global.

El comercio electrónico es un asunto de gran relevancia en la actualidad ya que ayuda a romper la barrera de la distancia en sistemas de mercado en un mundo que, para el caso, se ha convertido en una aldea global que está al alcance de todos, por el hecho de que el uso de estas tecnologías se enseña desde los primeros años, lo que provoca ver el uso de estas herramientas de manera natural en los negocios, gobiernos, hogares (CONCANACO, 2017: p. 47).

La resistencia al cambio es grande, y el mayor freno es el cultural. Muchos pequeños empresarios no entienden todavía las ventajas que implican las tecnologías de información. Se requiere tan sólo de un cambio de mentalidad para entrar al comercio electrónico y desarrollar las actividades empresariales, lo cual se traducirá en menores costos y mayor eficiencia operativa. [5]. 


\section{Plan de mercadotecnia como estrategia de marketing para la incursión de PyMEs al comercio electrónico}

Ante los conceptos de competencia global que empiezan a delimitar las estructuras de mercado, el plan de mercadotecnia es necesario para todas las empresas que busquen permanecer en él y proyectar un crecimiento sostenido que les permita hacer frente a las variables de un mercado globalizado.

La planeación estratégica de cada una de las unidades estratégicas de negocios de la empresa, exige la definición de la misión de negocios, analizar las oportunidades y los riesgos externos, analizar las fortalezas y debilidades internas, formular metas, formular estrategias, formular e implementar programas, obtener retroalimentación y ejercer control (Kotler,2012: p.60). Por esta razón, uno de los elementos de mayor trascendencia en este proceso de planeación estratégica es el establecimiento del plan de mercadotecnia para alcanzar las metas. [6].

El plan de mercadotecnia se convierte en una herramienta indispensable para todas aquellas empresas que desean mantener una posición competitiva en el mercado. Tal es el caso de las PyMEs comerciales y de servicios, interesadas en conservar un posicionamiento estable en el mercado actual, punto importante para el desarrollo y crecimiento de la economía regional.

La estructura del plan de mercadotecnia requiere que los expertos responsables del área tengan una visión global, que tengan también una visión analítica y que tengan los elementos de creatividad necesarios para programar estrategias efectivas de mercadotecnia.

A continuación se propone la estructura que debe guiar el contenido de un Plan de Mercadotecnia, de acuerdo con Fernández y Kotler, que permitirá delinear las estrategias necesarias para la incursión al comercio electrónico de las PyMEs comerciales y de servicios (Fernández, 2011; Kotler, 2006).

a) Descripción de la empresa.

b) Análisis situacional de mercadotecnia actual.

c) Evaluación del negocio: Análisis FODA.

d) Definición de los objetivos mercadológicos.

e) Desarrollo de las estrategias y tácticas mercadológicas.

f) Calendarización de actividades estratégicas y tácticas definidas.

g) Presupuestación de mercadotecnia.

h) Diseño de medidas de supervisión, evaluación y control.

\section{a. Descripción de la empresa}

Dentro de esta etapa de descripción del negocio, se establecerán las condiciones necesarias para realizar nuestro plan de mercadotecnia (Fernández, 2011: p. 32). Esta descripción del negocio, es un resumen ejecutivo, el cual indica de manera breve un panorama general de la empresa para una rápida revisión administrativa (Kotler, 2006: 104), por ello es importante que contenga: el tipo de organización en la cual nos encontramos, sus antecedentes y forma de operación; la misión de la empresa, que se refiere a la forma en que está constituida, su esencia misma y la relación de esta con su contexto social (Fernández, 2001: p. 38); la visión de la empresa, que nos indica cual es la meta que la compañía persigue a largo plazo, incluye la forma en que ésta se conceptualiza a sí misma en la actualidad y en el futuro; y la filosofía de la empresa, que se refiere a la forma en que ésta trabaja, la comunicación y espíritu que existe en la misma y la adopción de valores que trata de transmitirse a los empleados (Fernández, 2011: p. 41).

\section{b. Análisis situacional de mercadotecnia actual}

Es importante analizar los componentes del ambiente situacional en el cual opera nuestra empresa. Este análisis presenta la información más relevante sobre el mercado, producto, competencia, distribución y macroambiente. Para ello, tomamos como partida cinco aspectos importantes (Kotler, 1996: p.p. 104106): Situación del mercado, Situación del producto, Situación competitiva, Situación de la distribución y Situación del macroambiente.

\section{c. Evaluación del negocio: Análisis FODA}

Una vez descrita y analizada la situación actual de la empresa, es tiempo de hacer una evaluación del negocio, la cual consiste en aplicar una matriz FODA, que nos ayude a tomar decisiones en la formulación de la estrategia de mercadotecnia.

Ahora, se deben identificar las principales oportunidades/riesgos, fuerzas/vulnerabilidades, y aspectos particulares o alternativas que enfrenta la empresa. (Kotler, 2006: p. 106). El análisis comparativo de la matriz FODA representa en forma resumida las conclusiones de toda la evaluación del negocio, y se integra por cuatro variables: Fortalezas, Oportunidades, Debilidades y Amenazas.

Una vez analizados los cuatro factores de la matriz FODA, la empresa debe aplicar las situaciones descubiertas y definir las principales alternativas a las que debe dirigirse el plan (Kotler, 2006: p. 107).

\section{d. Definición de los objetivos mercadológicos}

Partiendo del análisis de la situación, la empresa debe plantear sus objetivos, clasificarlos y establecer metas 
y un programa para lograrlas. La empresa debe fijar estos objetivos con respecto a los socios o accionistas, la reputación de la compañía, la tecnología y otros asuntos de interés (Kotler, 2013: 114).

\section{e. Desarrollo de las estrategias y tácticas mercadológicas}

Las metas y objetivos de marketing se pueden alcanzar por varios caminos. El trabajo en el desarrollo de estrategias de mercadotecnia consiste en elegir el modo de acción más efectivo con la finalidad de alcanzar o lograr la consecución de los objetivos (Kotler, 2013: p. 114).

En sí, el desarrollo de las estrategias de mercadotecnia, presentan el enfoque mercadotécnico general que se utilizará para lograr los objetivos del Plan de Mercadotecnia (Kotler: 2006: p. 104), en otras palabras, por el mismo Kotler, "la estrategia es el camino elegido para conseguir un objetivo..." (Kotler, 2013: p. 37).

Al diseñar las estrategias de mercadotecnia, se deben desarrollar las tácticas correspondientes para el cumplimiento de éstas. Las tácticas deben contemplar todas las actividades que se requiere desarrollar para llevar a cabo la estrategia, de tal forma que se contemplen en su totalidad las variables que afectan la misma. [7]

\section{f. Calendarización de actividades estratégicas y tácticas definidas}

Todas y cada una de las estrategias deben ser contempladas en el itinerario de actividades de mercadotecnia; sin embargo, es posible realizar calendarios independientes, de tal forma que se tenga uno de medios, otro de promociones, otro de estudios de mercado.

Otro punto importante que debe contener el calendario, aparte de indicar las acciones a desarrollar, es señalar las personas concretas que van a llevar a cabo el plan (Kotler, 2013: p. 114).

No es estrictamente necesario el incluir todas y cada una de las tácticas en el itinerario de actividades, ya que las mismas lo harían sumamente complejo; sin embargo, es conveniente realizar calendarios alternos que permitan tener presentes a cada una de estas tácticas, de modo tal que puedan cumplirse con oportunidad (Fernández, 2011: p. 195).

\section{g. Presupuestación de mercadotecnia}

Al establecer el presupuesto de mercadotecnia es necesario atender a las siguientes consideraciones (Fernández, 2011: p.p. 206-208):
1) Tiene que responder a una razón lógica de ventas; esto quiere decir que, los recursos asignados en el presupuesto deben generar un incremento en las ventas, en el corto, mediano o largo plazo;

2) El presupuesto responde a los programas estratégicos; por lo que no deben existir partidas presupuestales que no tengan relación directa con los programas estratégicos que fueron planeados;

3) La decisión presupuestal debe estar a cargo del área responsable; esto se refiere a que cada una de las áreas debe decidir su presupuesto;

4) A mayor presupuesto mayor responsabilidad y resultados esperados; esto es, que los resultados esperados van en función directa del monto del presupuesto establecido.

\section{h. Diseño de medidas de supervisión, evaluación y control}

La última sección del Plan de Mercadotecnia describe los controles para dar seguimiento a sus avances (Kotler, 2006: p. 109). Por ello, la única forma en que será posible cumplir con las estrategias de mercadotecnia de manera eficiente, es estableciendo medidas de control que permitan verificar el correcto desarrollo de las mismas (Fernández, 2011: p. 214).

La supervisión busca el establecer mecanismos correctivos inmediatos, que proporcionen a las actividades de mercadotecnia la seguridad necesaria para su ejecución. Es importante que cada uno de los involucrados en la realización de las actividades de mercadeo, realice actividades de supervisión que le permitan asegurar el éxito de las mismas (Fernández, 2011: p. 216).

La evaluación del plan de mercadotecnia inicia desde el momento en que se hace una evaluación del negocio, ya que de la misma dependerá la correcta estructura de los objetivos y estrategias del plan, después, se evalúan los objetivos, su coherencia, factibilidad y proyección, de esa misma forma todas y cada una de las actividades se deben ir evaluando para verificar si los planes pueden ser logrados (Fernández, 2011: p. 216).

\section{Conclusiones}

Las PyMEs comerciales y de servicios, se están enfrentando actualmente a cambios económicos muy acelerados. El establecimiento de plazas y centros comerciales las impulsa a desarrollar estrategias de negocios que les permitan mantener un posicionamiento sólido en el mercado, mismo que 
garantizará la competitividad que el país requiere en términos de globalización.

Con respecto a su desarrollo económico, existen diversos programas que apoyan a las PyMEs en su modernización, competitividad y gestión administrativa. Dichos programas han sido implantados, en su mayoría, por el gobierno federal por medio de instituciones como Nacional Financiera, el Banco de Comercio Exterior y la Secretaría de Economía, para brindar apoyo a este sector y hacer más competitivas a las empresas, destinando recursos para que los nuevos empresarios puedan poner en marcha sus proyectos e incrementar su productividad.

\section{Referencias}

[1] AMECE, Asociación Mexicana de Estándares para el Comercio Electrónico. Consultado en julio de 2019, en http://www.amece.org.mx/amece/ .

[2] AMIPCI. Asociación Mexicana de Internet (2017). "Estudio AMIPCI de Comercio Electrónico en México 2017". Consultado en julio de 2019, en http://www.amipci.org.mx/estudios.php.

[3] Fernández Valiñas, R. (2011). Manual para elaborar un plan de mercadotecnia. Un enfoque latinoamericano. México: ECAFSA, Thomson Learning

[4] INEGI. (2016). Micro, pequeña, mediana y grande empresa. Estratificación de los establecimientos. México: Censos económicos 2014

[5] Kotler, P. (2006). Dirección de la Mercadotecnia. Análisis, planeación, implementación y control. México: Prentice Hall Hispanoamericana.

[6] CONCANACO SERVYTUR, ITESM. (2017). Estrategias y Políticas Públicas para el Desarrollo Empresarial y la Competitividad. Una propuesta del sector comercio, servicios y turismo 2007-2012. México: Fondo de Apoyo para la Micro, Pequeña y Mediana Empresa.

[7] Rodríguez, G. (2015). "Comercio electrónico, una revisión desde la Unión Internacional de Telecomunicaciones". Revista de Derecho, Fundación Universidad del Norte. Barranquilla, Colombia. 